\title{
Workshop on Active Mining (AM-2004)
}

The workshop on Active Mining (AM-2004) was held on June 1, 2004 at Ishikawa Kousei Nenkin Kaikan in Kanazawa City, Japan, as a part of the Eighteenth Annual Conference of the Japanese Society for Artificial Intelligence (JSAI-2004). This is the third workshop that focuses on Active Mining; the first one was held on December 9, 2002, as a part of the Second IEEE International Conference on Data Mining (ICDM'02), and the second one was held on October 28, 2003 as a part of the 14th International Symposium on Methodologies for Intelligent Systems, both at Maebashi TERRASA, Maebashi City, Japan.

Active mining is a new direction in the knowledge discovery process for realworld applications handling various kinds of data with actual user need.

Our ability to collect data has been increasing at a dramatic rate, which we call information flood. However, our ability to analyze and understand massive data lags far behind our ability to collect them. The value of data is no longer in how much of it we have. Rather, the value is in how quickly and effectively can the data be reduced, explored, manipulated and managed.

For this purpose, Knowledge Discovery in Databases (KDD) emerges as a technique that extracts implicit, previously unknown, and potentially useful information (or patterns) from data. However, recent extensive studies and realworld applications show that the following requirements are indispensable to overcome information flood: 1) identifying and collecting the relevant data from a huge information search space (active information collection), 2) mining useful knowledge from different forms of massive data efficiently and effectively (usercentered active data mining), and 3) promptly reacting to situation changes and giving necessary feedback to both data collection and mining steps (active user reaction).

Active mining is proposed as a solution to these requirements, which collectively achieves various mining needs. By collectively achieving we mean that the total effect outperforms the simple add-sum effect that each effort can bring.

The objective of this workshop was to gather researchers as well as practitioners who are working on various research fields of active mining, share hardlearned experiences, and shed light on future development of active mining. This workshop addressed many aspects of active mining ranging from theories, methodologies, algorithms, to their applications. Through this workshop, we produced modern solutions facilitating data collection, processing and knowledge discovery and created synergy among different branches.

This book contains 8 papers selected from 11 papers presented in the workshop. We would like to thank all the authors who have submitted papers, with whom the workshop was possible.

July 2005

Masayuki Numao, Takahira Yamaguchi, Shusaku Tsumoto and Hiroshi Motoda 